\title{
Disseminated and Hyperinfection Fatal Strongyloidiasis in Two Patients on Immunosuppressive Therapy: A Case Report
}

\author{
Ahmad Reza Meamar (PhD) \\ Department of Parasitology and \\ Mycology, School of Medicine, Iran \\ University of Medical Sciences, \\ Tehran, Iran \\ Nahid Jalallou (PhD) \\ Department of Medical Laboratory \\ Science, AJA University of Medical \\ Sciences, Tehran, Iran \\ Leila Masoori \\ Department of Parasitology and \\ Mycology, School of Medicine, Iran \\ University of Medical Sciences, \\ Tehran, Iran \\ Maryam Alipour \\ Department of Parasitology and \\ Mycology, School of Medicine, Iran \\ University of Medical Sciences, \\ Tehran, Iran \\ Corresponding author: Nahid \\ Jalallou \\ Tel: +989122877110 \\ Email: nahidjalallou@yahoo.com
}

Address: Iran University of Medical Sciences, Tehran, Iran

Received: 11 Nov 2018

Revised: 02 May 2019

Accepted: 08 May 2019

\section{(c) (i) (8)}

This work is licensed under a Creative Commons Attribution 4.0 License.

\section{ABSTRACT}

Background and 0bjective: Strongyloides infection is usually asymptomatic in healthy individuals; however, immunocompromised patients are subject to potential hyperinfection involvement. Hereby, we report disseminated and hyperinfection strongyloidiasis in two immunosuppressed patients from north of Iran, an endemic region for Strongyloides stercoralis.

Case description: After assessment of clinical symptoms, disseminated strongyloidiasis was identified in the first case with pemphigus vulgaris by the presence of abundant larvae in direct stool smears and gastric biopsy. The second patient had metastatic carcinoma and was hospitalized with complicated symptoms. The infection was diagnosed by detection of numerous first-stage larvae in wet mount stool smears and agar plate analysis. Despite antiparasitic treatment, both patients died during the hospitalization.

Conclusion: Delayed diagnosis of active strongyloidiasis in immunosuppressed patients can lead to hyperinfection syndrome. Thus, screening for $S$. stercoralis infection in these patients is recommended in endemic regions to reduce mortalities.

Keywords: Strongyloides stercoralis, Disseminated strongyloidiasis, Hyperinfection.

This paper should be cited as: Meamar AR, Jalallou N, Alipour M [Disseminated and Hyperinfection Fatal Strongyloidiasis in Two Patients on Immunosuppressive Therapy: A Case Report]. mljgoums. 2019; 13(6): 51-54 


\section{INTRODUCTION}

Strongyloidiasis is a patristic infection caused by Strongyloides stercoralis. It is highly distributed in tropical and subtropical regions with poor sanitary conditions. The prevalence range is estimated to be about 50 to 100 million cases worldwide (1).

The infective filariform larvae that exist in soil can penetrate through skin and reach the lungs via circulation. After two molts, the worms reach the small intestine where they mature and lay eggs, which hatch as rhabditoid larvae that are excreted in feces (2). These larvae can also mature into filariform larvae within the gastrointestinal tract and infect intestinal tissue or penetrate perianal skin and enter the circulatory system, resulting in "autoinfection" $(1,2)$.

North of Iran is considered an endemic area for strongyloidiasis where most cases are identified among rice field and tea garden workers. Following autoinfection, $S$. stercoralis can remain stable within the host for years or decades (3).

Although infection is frequently accompanied by mild gastrointestinal symptoms in healthy people, uncontrolled reproduction of the parasite (hyperinfection syndrome, HS) and/or dissemination of larvae (disseminated strongyloidiasis, DS) to all internal organs in immunocompromised individuals can have various manifestations and risk of death (4). In this article, we describe two cases of disseminated and hyperinfection strongyloidiasis in immunosuppressed patients from north of Iran, an endemic region for strongyloidiasis.

\section{CASE PRESENTATION}

\section{Case 1}

A 57-year-old man from Tonekabon (Mazanderan Province, north of Iran) with a history of pemphigus vulgaris was hospitalized in the Chamran Medical Center in Tehran, Iran. His symptoms included abdominal pain, cough, sputum discharge, chest pain, weakness, dizziness and anemia. He had been taking prednisolone for several years and ciprofloxacin for two months. The initial laboratory results at the time of admission are shown in Table 1.

Numerous rhabditiform larvae of $S$. stercoralis were identified in direct smear and formalinether examination of diarrheic stool samples. $S$. stercoralis hyperinfection was diagnosed after detection of rhabditiform and filariform larvae in sputum smears and agar plate stool culture. Abdominal sonography and computed tomography scan were normal.

Massive gastrointestinal hemorrhage was observed and endoscopy revealed severe duodenal inflammation and scattered sympathetic stomach ulcers. Numerous $S$. stercoralis larvae were observed in the gastric biopsy (Figure 1). Subsequently, the patient developed acute respiratory distress syndrome and received ventilatory support. Albendazole therapy $(400 \mathrm{mg})$ twice daily for three days had no curative effect. The case died of cardiac arrest at the initiation of ivermectin therapy.

Table 1- Initial laboratory results of case 1 at the time of admission.

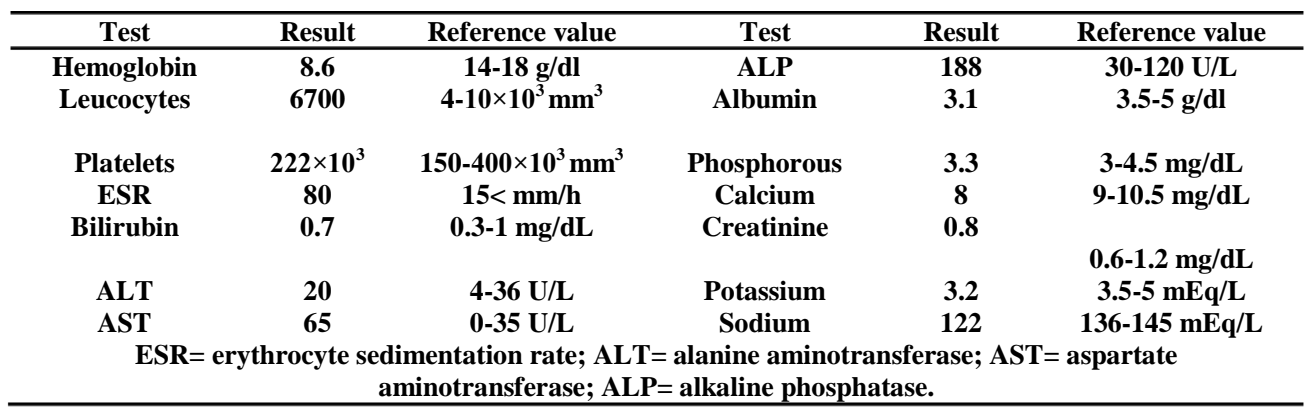

Case 2

A 45-year old man from Amol (Mazandaran Province, north of Iran) had been suffering from pain in the waist three years before admission to Imam Reza Hospital in Tehran, Iran. Sonography revealed a tumor on the left kidney and the patient was ultimately diagnosed with renal cell carcinoma. Following nephrectomy of the left kidney, the patient was treated with interferon alpha and vincristine for two years. The tumor had metastasized to the lungs and bone marrow six months after the last chemotherapy treatment. 
Physical examination revealed hydronephrosis and osteomyelitis. The patient also developed diffuse abdominal discomfort with irregular gastric pain, nausea and diarrhea, but no lesion or ulceration was identified. Table 2 shows the laboratory results at the last time of hospitalization.

In fresh fecal samples, abundant first-stage rhabditiform larvae were detected by wet mount and formalin-ether assessment.
Moreover, various rhabditiform and filariform $S$. stercoralis larvae were identified on agar plate cultures. The patient was placed on albendazole $(400 \mathrm{mg}$ ) twice daily for three days. Despite the albendazole therapy, respiratory failure ensued; therefore, the patient was intubated and transferred to the intensive care unit. The clinical conditions worsened and he died five days after diagnosis of strongyloidiasis.

Figure 1-Hematoxilin and eosin staining of biopsy samples from case 1. A: Numerous larvae of Strongyloides stercoralis in gastric section (100× magnification). B: High power field image, showing fine points of larvae $(400 \times$ magnification).

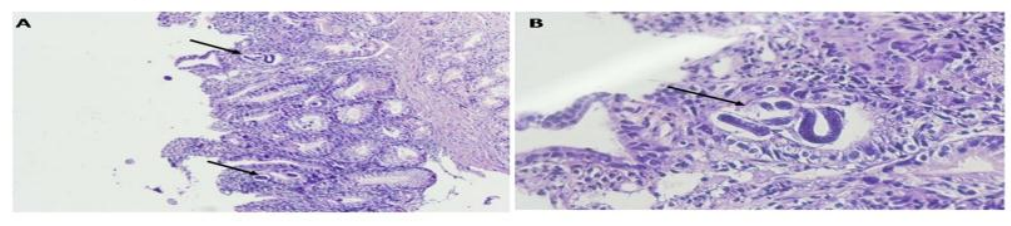

Table 2- Initial laboratory results of case 2 at the last time of hospitalization.

\begin{tabular}{|c|c|c|c|c|c|}
\hline Test & Result & $\mathbf{R V}$ & Test & Result & $\mathbf{R V}$ \\
\hline Hemoglobin & 10.3 & $14-18 \mathrm{~g} / \mathrm{dl}$ & ALP & 109 & $30-120 \mathrm{U} / \mathrm{L}$ \\
\hline Leucocytes & $32.3 \times 10^{3}$ & $4-10 \times 10^{3} \mathrm{~mm}^{3}$ & Albumin & 3.2 & $3.5-5 \mathrm{~g} / \mathrm{dl}$ \\
\hline Platelets & $228 \times 10^{3}$ & $150-400 \times 10^{3} \mathrm{~mm}^{3}$ & Phosphorous & 4.3 & $3-4.5 \mathrm{mg} / \mathrm{dl}$ \\
\hline ESR & 109 & $15<\mathrm{mm} / \mathrm{h}$ & Calcium & 9.8 & $9-10.5 \mathrm{mg} / \mathrm{dl}$ \\
\hline Bilirubin & 1.1 & $0.3-1 \mathrm{mg} / \mathrm{dL}$ & Creatinine & 1.4 & $0.6-1.2 \mathrm{mg} / \mathrm{dl}$ \\
\hline ALT & 32 & 4-36 U/L & Potassium & 3.2 & 3.5-5 mEq/L \\
\hline AST & 42 & 0-35 U/L & Sodium & 139 & $136-145 \mathrm{mEq} / \mathrm{L}$ \\
\hline
\end{tabular}

\section{DISCUSSION}

Although strongyloidiasis is endemic in north of Iran, the reported prevalence is $0.3 \%$ in the general population (3-5). Due to the moist climate and inhabitants' outdoor occupations, north of Iran is a suitable for $S$. stercoralis survival and subsequent human infection (3).

Various immunosuppressive conditions such as organ transplantation, immunosuppressant therapy, cancer, acquired immunodeficiency syndrome, human T-cell leukemia virus type 1 infection, malnutrition and collagen diseases are more specifically associated with the transformation of chronic strongyloidiasis into hyperinfection (6). To our knowledge, this is the first report of HS in a renal cell carcinoma patient. Although HS has been reported in pemphigus vulgaris patients $(7,8)$, only one case of HS in these patients has been reported from north of Iran (9). Our first case had taken prednisolone for several years. Corticosteroid therapy in strongyloidiasis patients remains the most frequent cause of HS $(4,6)$. The possible effects of duration and dosage of corticosteroid therapy on strongyloidiasis are not known (10). Probably, insufficient humoral and cellular immune response leads to rhabditiform transformation into filariform larvae, subsequently replication in the gastrointestinal tract (HS) and migration to the pulmonary system or other organs (DS)

increase. (11). Moreover, it has been proposed that glucocorticoids might have stimulatory effect on ecdysteroid (molting hormones) production or on the adult female's ability to produce eggs $(6,12)$. The HS-associated mortality rate is $15 \%$ but may increase to $87 \%$ with dissemination (12).

Our second case was treated with vincristine. Several hyperinfection cases have been reported due to vincristine treatment (13). It has been suggested that vinca alkaloids are toxic to myenteric neurons, thereby decreasing intestinal motility and increasing the amount of time that rhabditiform larvae have to molt to 
invasive filariform larvae (6). Clinical features of strongyloidiasis are not well-defined. In addition, laboratory diagnosis of chronic infection is difficult due to the irregular release of few larvae in stool. In contrast, direct examination of stool specimens can be useful in HS patients (13). The agar plate method is a sensitive technique for strongyloidiasis detection (2). In our cases, infections were diagnosed from clinical signs and symptoms. Moreover, they were confirmed by observation of first-stage larvae in direct smears and unusual loads of filariform larvae on agar plates.

Although eosinophilia is common in helminthic diseases, it could be normal in immunocompromised patients with severe strongyloidiasis (4). However, corticosteroids can reduce the number of circulating

\section{REFERENCES}

1. Toledo R, Muñoz-Antoli C, Esteban JG. Strongyloidiasis with emphasis on human infections and its different clinical forms. Adv Parasitol. 2015; 88: 165241. doi: 10.1016/bs.apar.2015.02.005.

2. Buonfrate D, Formenti F, Perandin F, Bisoffi Z. Novel approaches to the diagnosis of Strongyloides stercoralis infection. Clin Microbiol Infect. 2015; 21(6): 543-52. doi: 10.1016/j.cmi.2015.04.001.

3. Rokni MB. The present status of human helminthic diseases in Iran. Ann Trop Med Parasitol. 2008; 102(4): 283-95. doi: 10.1179/136485908X300805.

4. Geri G, Rabbat A, Mayaux J, Zafrani L, ChalumeauLemoine L, Guidet B, et al.

Strongyloides stercoralis hyperinfection syndrome: a case series and a review of the literature. Infection. 2015; 43(6): 691-8. doi: 10.1007/s15010-015-0799-1.

5. Tabei SZ, Asadian F, Fakhar M, Safaei A. Gastrointestinal hyperinfection due to Strongyloides stercoralisin a patient with Behcet's syndrome. Comp Clin Pathol. 2009; 18: 89-91. DOI:10.1007/s00580-0080750-2.

6. Keiser PB, Nutman TB. Strongyloides stercoralis in the immunocompromised population. Clin Microbiol Rev. 2004; 17(1): 208-217. doi: 10.1128/CMR.17.1.208217.2004

7. Reddy IS, Swarnalata G. Fatal disseminated strongyloidiasis in patients on immunosuppressive therapy: Report of two cases. Indian J Dermatol Venereol Leprol. 2005; 71(1): 38-40 by enhancing apoptosis of T-helper 2 cells and preventing eosinophil

\section{CONCLUSION}

Based on the observations, the delayed diagnosis of active strongyloidiasis in immunosuppressed patients can lead to HS. Thus, screening for $S$. stercoralis infection in these patients is recommended in endemic regions to reduce mortalities.

\section{ACKNOWLEDGMENTS}

The present work has been supported financially by AJA University of Medical Sciences (Grant No. 59082) and ethics code: IR.AJAUMS.REC.1396.116.

\section{CONFLICT OF INTEREST} interest.

The authors declare no conflict of

8. Sarubbi FA. Hyperinfection with Strongyloides during treatment of pemphigus vulgaris. Arch Dermatol. 1987; 123(7): 864-5. doi:10.1001/archderm.1987.01660310028005.

9. Fakhar M, Gholami Z, Banimostafavi ES, Madjidi H. Respiratory hyperinfection caused by Strongyloides stercoralis in a patient with pemphigus vulgarisand mini review on diagnosis and treatment of strongyloidiasis. Comp Clin Pathol. 2010; 19(6): 621-625. doi: 10.1007/s00580-010-0995-4.

10. Buonfrate D, Requena-Mendez A, Angheben A, Muñoz J, Gobbi F, Van Den Ende J, et al. Severe strongyloidiasis: a systematic review of case reports. BMC Infect Dis. 2013; 13: 78. doi: 10.1186/1471-2334-13-78.

11. Concha R, Harrington Jr W, Rogers Al. Intestinal strongyloidiasis: recognition, management, and determinants of outcome. J Cli Gastroenterol. 2005; 39(3): 203-211.

12. Vadlamudi RS, Chi DS, Krishnaswamy G. Intestinal strongyloidiasis and hyperinfection syndrome. Clin Mol Allergy. 2006; 4: 8. doi: 10.1186/1476-7961-4-8.

13. Weatherhead JE, Mejia R. Immune Response to Infection with Strongyloides stercoralis in patients with infection and hyperinfection. Curr Trop Med Rep. 2014; 1(4): $229-233$ 